\title{
Novel Coronavirus Polymerase and Nucleotidyl-transferase Structures: Potential to Target New Outbreaks
}

Wen-Fa Zhang ${ }^{\$}$, Preyesh Stephen ${ }^{\$}$, Jean-François Thériault ${ }^{+}$, Ruixuan Wang ${ }^{+}$and Sheng-Xiang Lin*

Axe Molecular Endocrinology and Nephrology, CHU Research Center and Laval University, Québec City, Québec G1V 4G2, Canada

\$: co-first authors

${ }^{+}$: authors contribute similarly to this work;

*: correspondence to: sxlin@ crchul.ulaval.ca

\section{Materials and Methods}

Model Building

Genome sequence of SARS-CoV and Wuhan novel Coronavirus (SARS-CoV-2) was obtained from NCBI. As the sequence of the nsp7, nsp8 and were not annotated in NCBI database, their sequence was determined by aligning the whole sequence of SARS-CoV-2 to SARS-CoV genome. Templates for modelling was determined by amino acid alignment of Protein Data Bank (PDB) by NCBI blast. The 3D model of SARS-CoV-2 nsp12 and RdRp was derived by template structure from SARS nsp12 (PDB ID: 6NUR) while nsp7 was derived by template structure from SARSCoV ns7 structure (PDB: 2AHM) by Modeller $9.23^{1}$. Nsp8 was modelled by swiss-model ${ }^{2}$ using SARS-CoV ns8 structure (PDB: 2AHM) as the tempplate.

Model Refinement and Upload

Pymol was used for the calculation of each root mean square deviation (RMSD) and viewing of the structure. The model was refined and energy minimized using SwissPDB viewer ${ }^{3}$. Validation of the structure was done by a series of tools including PROVE ${ }^{4}$, ERRAT ${ }^{5}$, VERIFY3D ${ }^{6}$ and PROCHECK ${ }^{7}$. The 3D-structures were available in Protein Model DataBase (PMDB, http://srv00.recas.ba.infn.it/PMDB/main.php) with reference PM0082694, PM0082704 and PM0082703 for SARS-CoV-2 nsp12, nsp7 and nsp8, respectively.

Virtual Screening

Docking of RdRp with ATP, Galidesivir, Favipiravir and Penciclovir was performed using CCDC GOLD. 10 Á around (x:145, y142, z155) was chosen as the binding pocket and a maximum of 10 docked conformers for each ligand was generated. Hydrophobic interaction or hydrogen bond interaction with RdRp was calculated by Ligplot ${ }^{9}$. 


\section{Reference}

1. Eswar, N.; Webb, B.; Marti-Renom, M. A.; Madhusudhan, M.; Eramian, D.; Shen, M. y.; Pieper, U.; Sali, A. Comparative protein structure modeling using Modeller. Current protocols in bioinformatics 2006, 15 (1), 5.6. 1-5.6. 30.

2. Waterhouse, A.; Bertoni, M.; Bienert, S.; Studer, G.; Tauriello, G.; Gumienny, R.; Heer, F. T.; de Beer, T. A. P.; Rempfer, C.; Bordoli, L. SWISS-MODEL: homology modelling of protein structures and complexes. Nucleic acids research 2018, 46 (W1), W296W303.

3. Guex, N.; Peitsch, M. C. SWISS-MODEL and the Swiss-Pdb Viewer: an environment for comparative protein modeling. electrophoresis 1997, 18 (15), 2714-2723.

4. Pontius, J.; Richelle, J.; Wodak, S. J. Deviations from standard atomic volumes as a quality measure for protein crystal structures. Journal of molecular biology 1996, 264 (1), 121-136.

5. Colovos, C.; Yeates, T. O. Verification of protein structures: patterns of nonbonded atomic interactions. Protein science 1993, 2 (9), 1511-1519.

6. Bowie, J. U.; Luthy, R.; Eisenberg, D. A method to identify protein sequences that fold into a known three-dimensional structure. Science 1991, 253 (5016), 164-170.

7. Laskowski, R. A.; MacArthur, M. W.; Moss, D. S.; Thornton, J. M. PROCHECK: a program to check the stereochemical quality of protein structures. Journal of applied crystallography 1993, 26 (2), 283-291.

8. O'Boyle, N. M.; Banck, M.; James, C. A.; Morley, C.; Vandermeersch, T.; Hutchison, G. R. Open Babel: An open chemical toolbox. Journal of cheminformatics 2011, 3 (1), 33.

9. Wallace, A. C.; Laskowski, R. A.; Thornton, J. M. LIGPLOT: a program to generate schematic diagrams of protein-ligand interactions. Protein engineering, design and selection 1995, 8 (2), 127-134. 
SARS-CoV-2 sequence used for modelling:

SARS-CoV-2 nsp12 (932 a.a., modelled sequence a.a. 117-895, Template: 6BUR.A)

SADAQSFLNRVCGVSAARLTPCGTGTSTDVVYRAFDIYNDKVAGFAKFLKTNCCRFQEKDEDD NLIDSYFVVKRHTFSNYQHEETIYNLLKDCPAVAKHDFFKFRIDGDMVPHISRQRLTKYTMADL VYALRHFDEGNCDTLKEILVTYNCCDDDYFNKKDWYDFVENPDILRVYANLGERVRQALLKTV QFCDAMRNAGIVGVLTLDNQDLNGNWYDFGDFIQTTPGSGVPVVDSYYSLLMPILTLTRALTAE SHVDTDLTKPYIKWDLLKYDFTEERLKLFDRYFKYWDQTYHPNCVNCLDDRCILHCANFNVLFS TVFPPTSFGPLVRKIFVDGVPFVVSTGYHFRELGVVHNQDVNLHSSRLSFKELLVYAADPAMHA ASGNLLLDKRTTCFSVAALTNNVAFQTVKPGNFNKDFYDFAVSKGFFKEGSSVELKHFFFAQDG NAAISDYDYYRYNLPTMCDIRQLLFVVEVVDKYFDCYDGGCINANQVIVNNLDKSAGFPFNKW GKARLYYDSMSYEDQDALFAYTKRNVIPTITQMNLKYAISAKNRARTVAGVSICSTMTNRQFHQ KLLKSIAATRGATVVIGTSKFYGGWHNMLKTVYSDVENPHLMGWDYPKCDRAMPNMLRIMAS LVLARKHTTCCSLSHRFYRLANECAQVLSEMVMCGGSLYVKPGGTSSGDATTAYANSVFNICQ AVTANVNALLSTDGNKIADKYVRNLQHRLYECLYRNRDVDTDFVNEFYAYLRKHFSMMILSDD AVVCFNSTYASQGLVASIKNFKSVLYYQNNVFMSEAKCWTETDLTKGPHEFCSQHTMLVKQGD DYVYLPYPDPSRILGAGCFVDDIVKTDGTLMIERFVSLAIDAYPLTKHPNQEYADVFHLYLQYIR KLHDELTGHMLDMYSVMLTNDNTSRYWEPEFYEAMYTPHTVLQ

SARS-CoV-2 nsp7 (83 a.a., modelled sequence a.a. 1-74, Template: 2AHM.B)

SKMSDVKCTSVVLLSVLQQLRVESSSKLWAQCVQLHNDILLAKDTTEAFEKMVSLLSVLLSMQ GAVDINKLCEEMLDNRATLQ

SARS-CoV-2 nsp8 (198 a.a., modelled sequence a.a. 1-191, Template: 2AHM.G)

AIASEFSSLPSYAAFATAQEAYEQAVANGDSEVVLKKLKKSLNVAKSEFDRDAAMQRKLEKMA DQAMTQMYKQARSEDKRAKVTSAMQTMLFTMLRKLDNDALNNIINNARDGCVPLNIIPLTTAA KLMVVIPDYNTYKNTCDGTTFTYASALWEIQQVVDADSKIVQLSEISMDNSPNLAWPLIVTALR ANSAVKLQ

SARS-COV-2 model root-mean-square deviation (RMSD).

\begin{tabular}{|l|l|l|}
\hline Structure & RMSD & PMDB ID \\
\hline nsp12 & 0.131 & PM0082694 \\
\hline nsp7 & 0.134 & PM0082704 \\
\hline nsp8 & 0.093 & PM0082703 \\
\hline
\end{tabular}




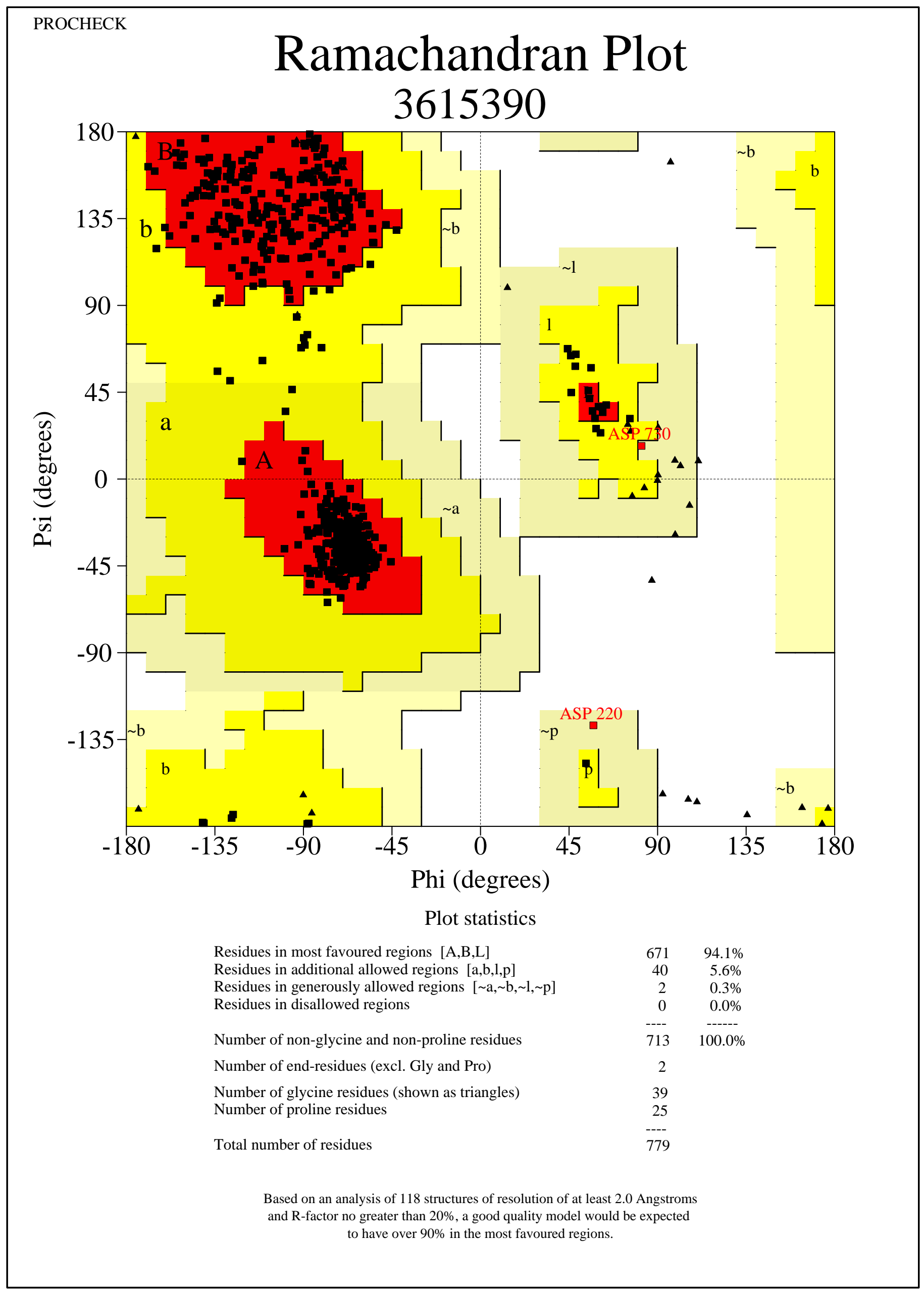




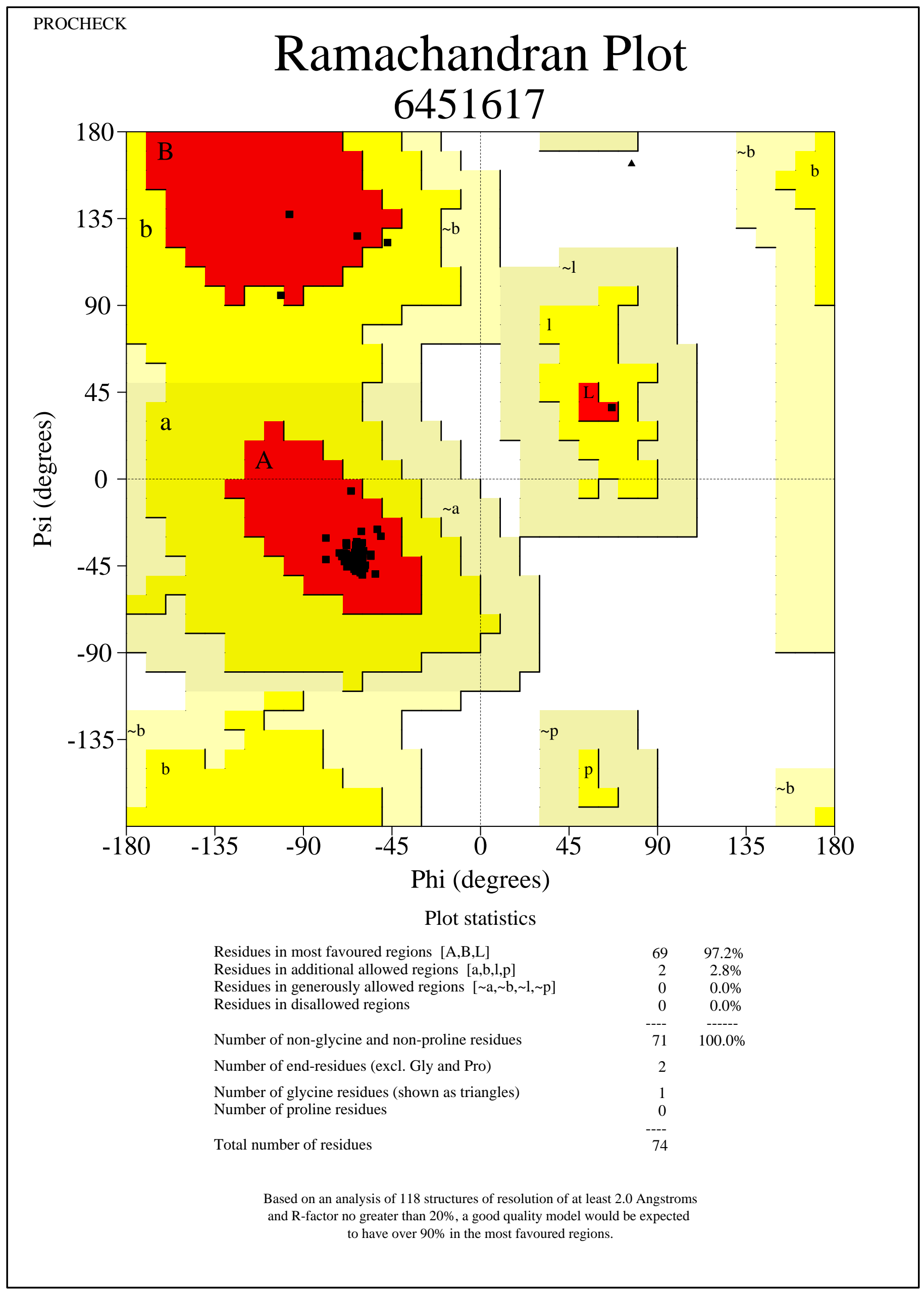




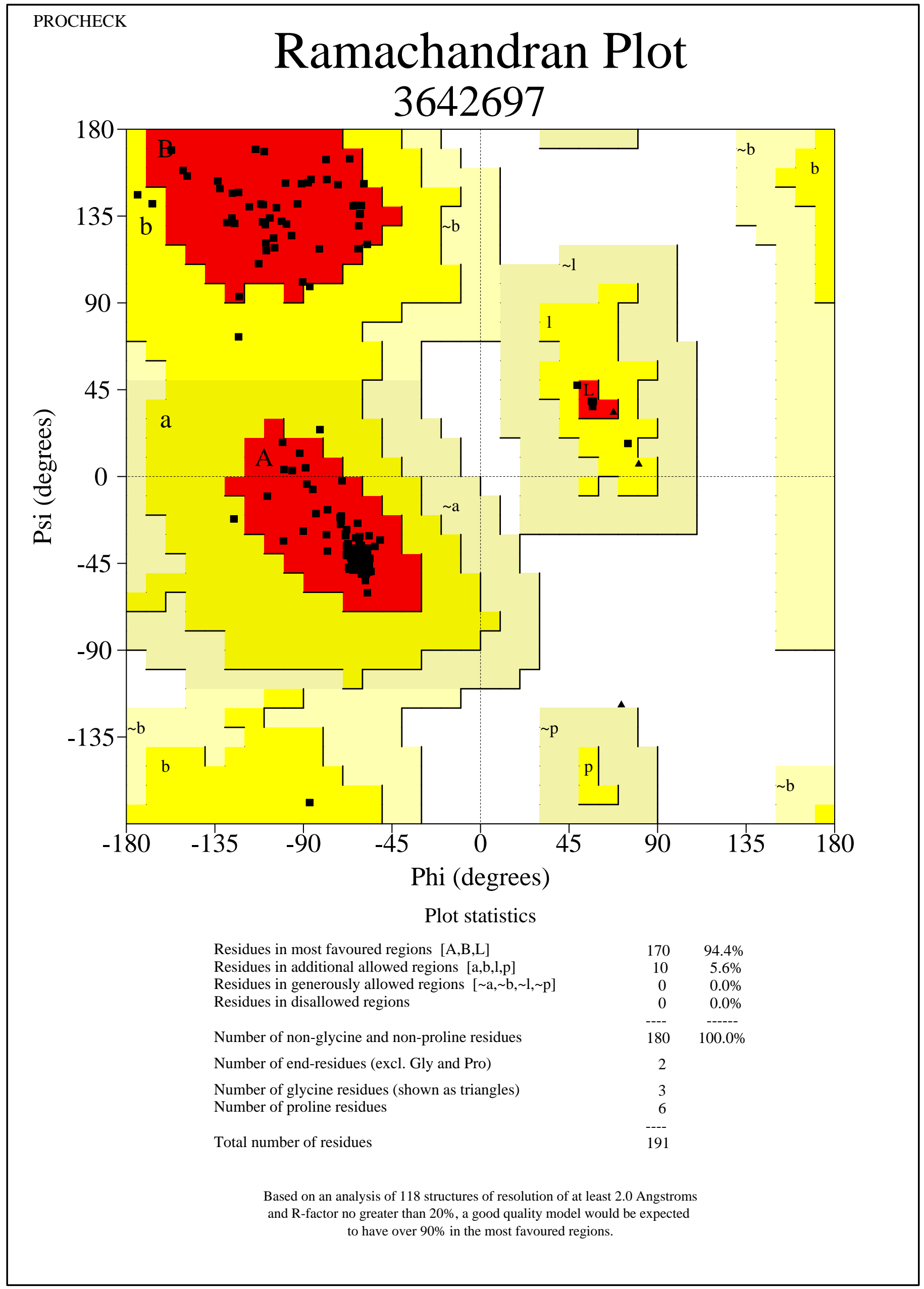


Hydrophobic and hydrogen bond interaction between drugs and RdRp

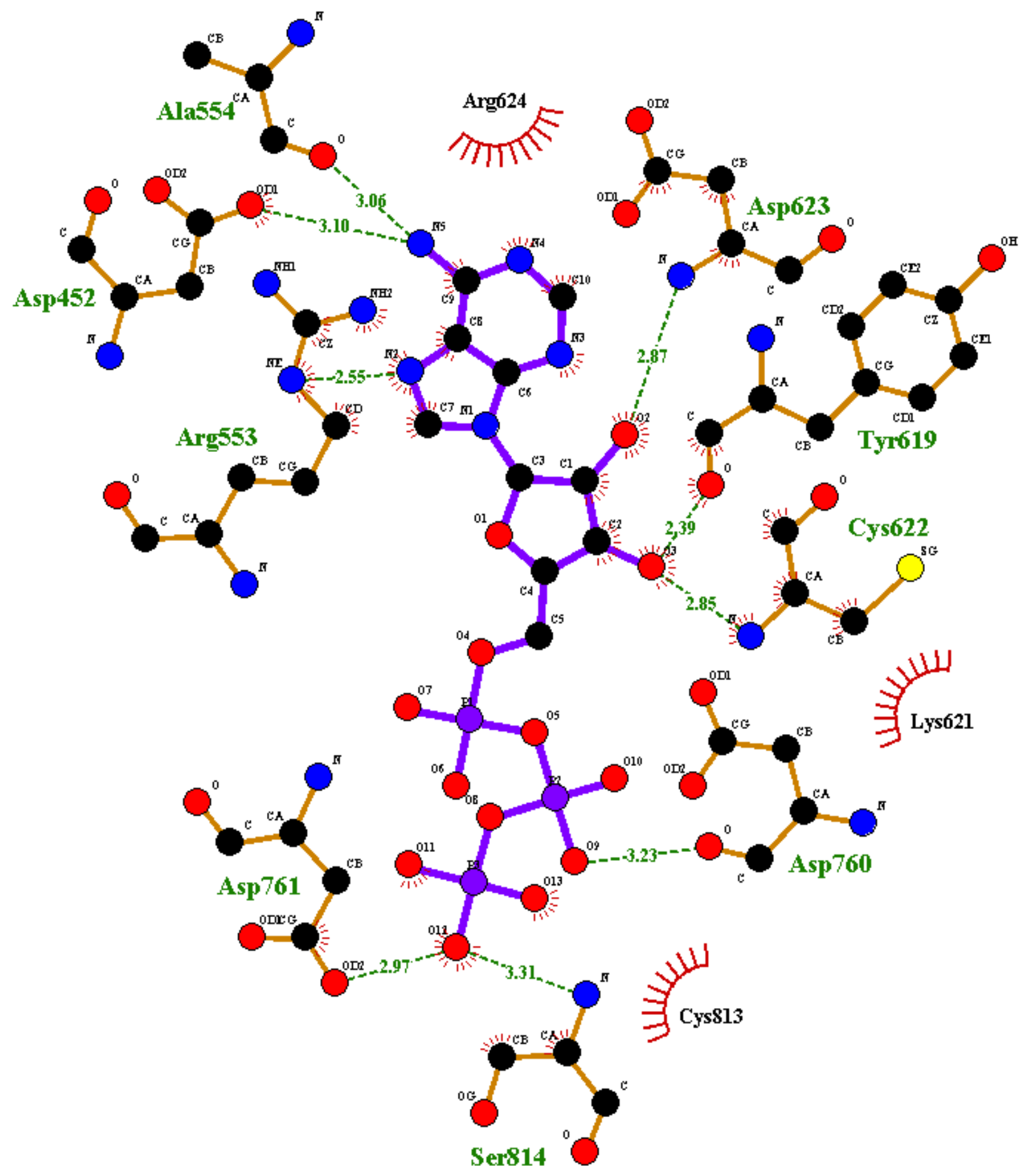

ATP 


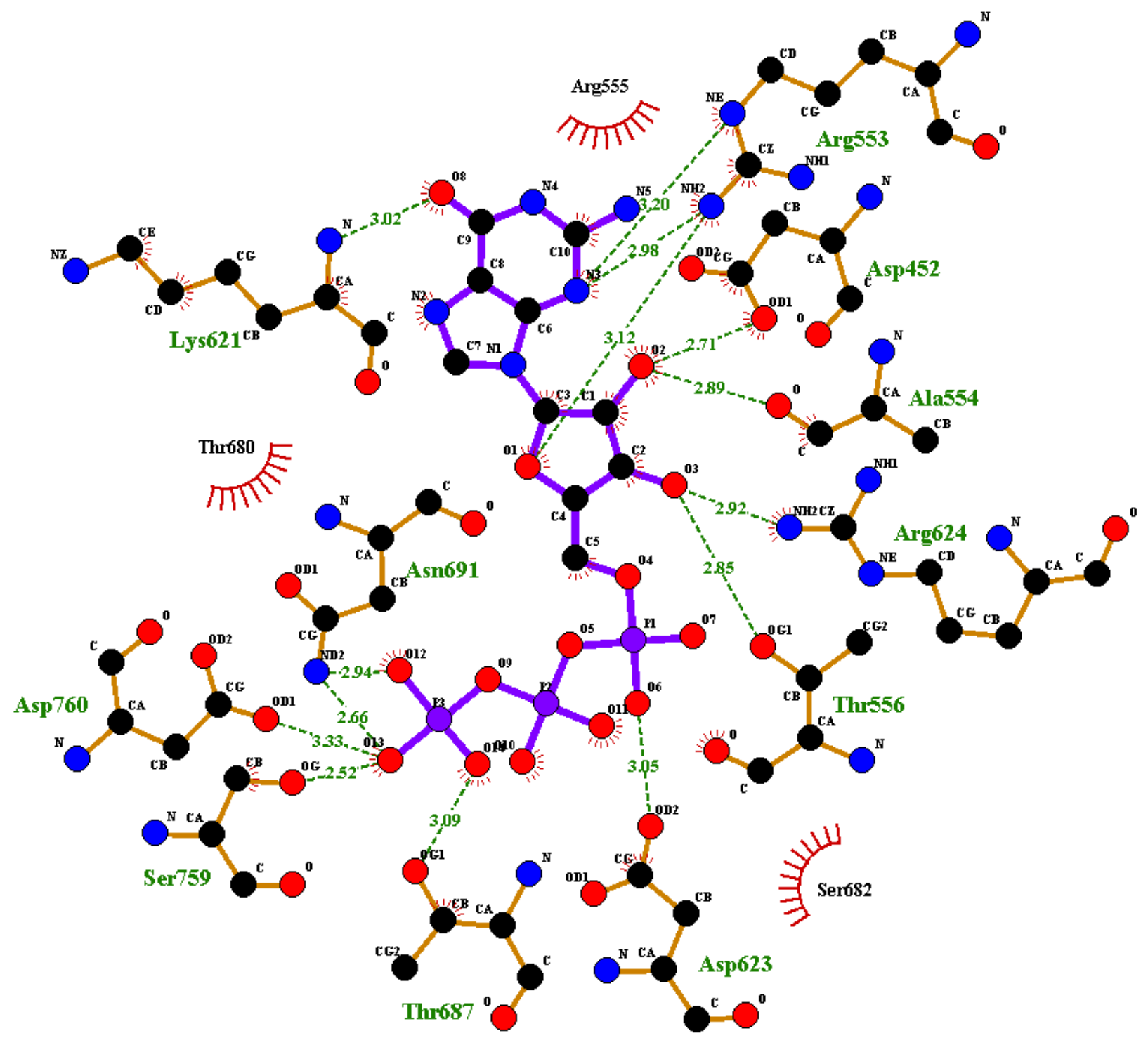

GTP 


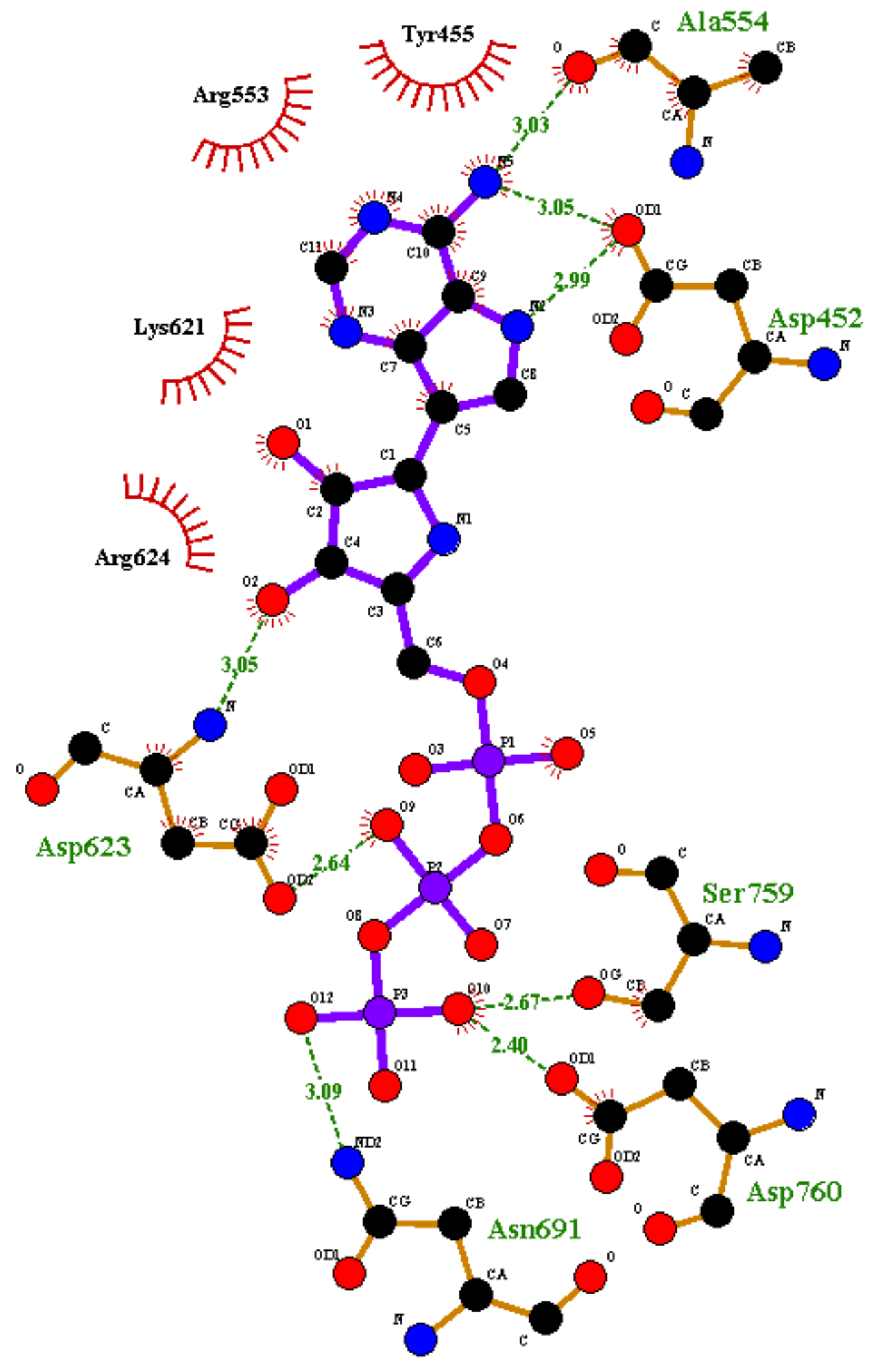

Galidesivir 


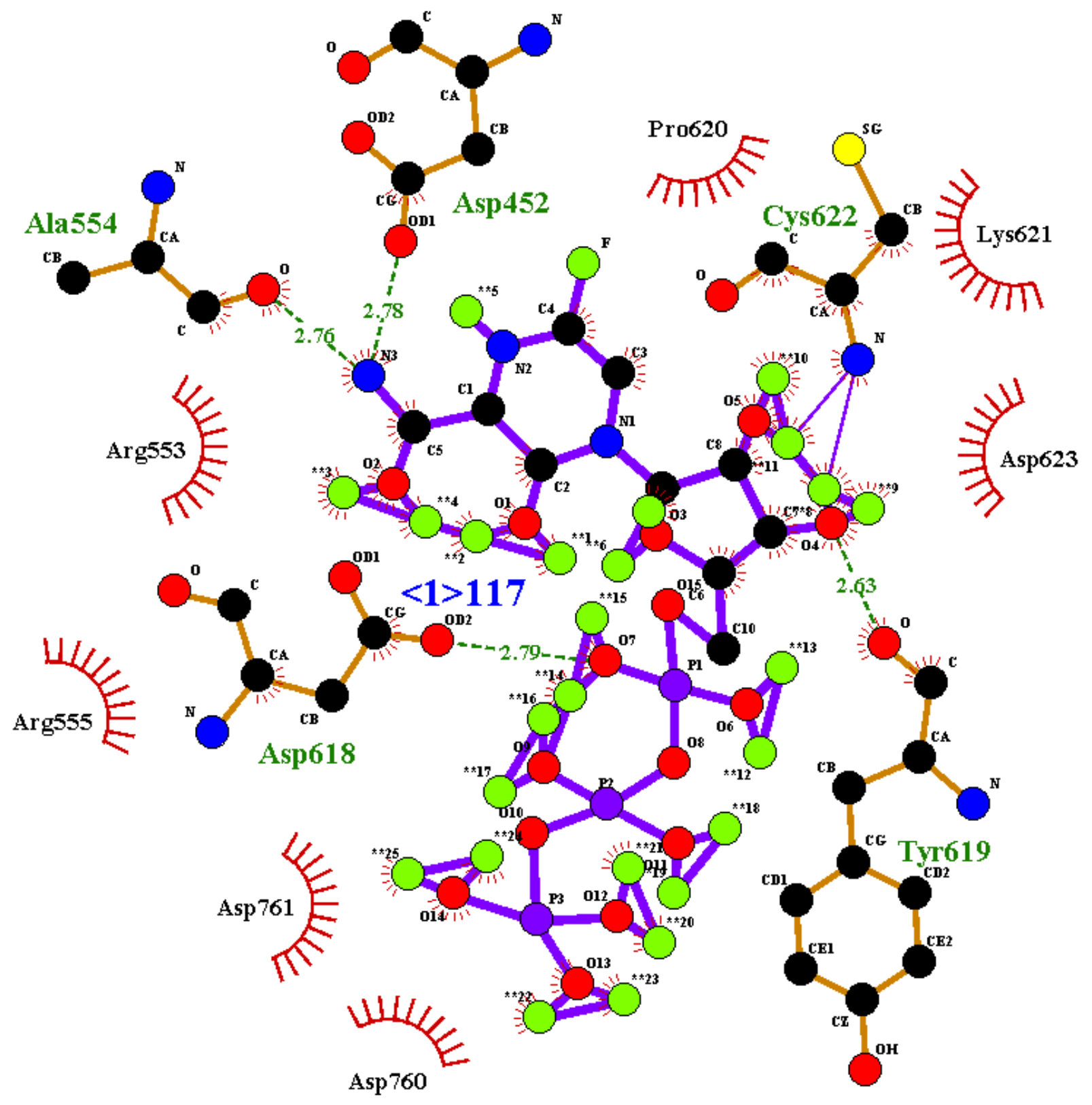

Favipiravir 


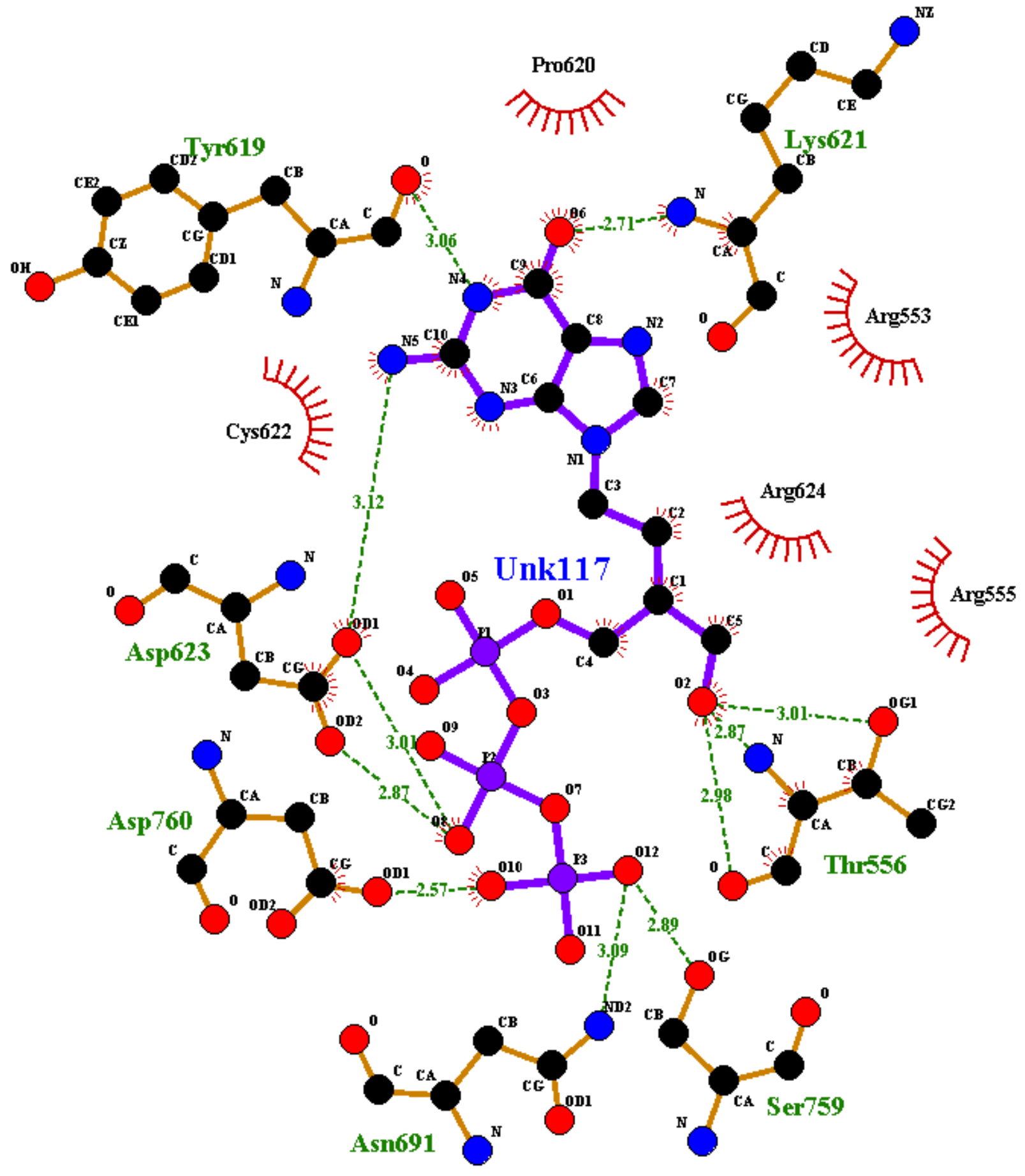

Penciclovir 